\title{
Teacher-as-reader: Oral feedback on ESL student writing
}

Lynn McAlpine

Described is an oral feedback procedure which teachers may choose to use if they want to respond to student writing not as editors and proofreaders but as interested first-language readers intent on coming to comprehend the written message.

There has been a realization in recent years that the process of writing, whether in a first, second or other language, is one of discovery in which writers increasingly clarify their thoughts through the writing of successive drafts (Flower and Hayes 1981; Raimes 1985; Chenoweth 1987). Yet, as language teachers we often respond to students' writing as if we were looking at final drafts and we act as editors or proofreaders by focussing on language-specific errors (Zamel 1985).

Granted, our tendency to focus on language-specific errors may be partly the result of constraints beyond our control, e.g., exams. Yet, if writing is a process of discovering and then communicating meaning, our response, at least in initial drafts, might be more helpful if it represented a first-language reader's attempt to make meaning of a text.

How does a reader draw meaning from a text? The reader of a text enters into a dialogue with the writer in which the interlocutors are removed in time and space from each other. Thus, the receiver of the message cannot negotiate the meaning directly with the sender as in an oral dialogue. Readers are forced to negotiate or interpret meaning by talking themselves through the document. And it is information regarding this process of interpretation that is of value to the writer in making the text more comprehensible. In what follows, I describe how to use a procedure, a think-aloud protocol, that gives this type of feedback to students on their writing ${ }^{1}$. Second, I describe how the student uses the feedback. Third, I describe the potential of this procedure as a tool for the ESL writing teacher. Fourth, I provide a sample of its use: a transcript of a think-aloud protocol with pre-think-aloud and post-think-aloud student drafts. Before proceeding, however, I will briefly describe the ESL students with whom I have used the procedure: adults already working in their second language, English, who are taking writing courses as they and/or their supervisors feel their writing skills need improving. 


\section{Using the think-aloud protocol to respond to student writing}

The think-aloud protocol is an easy procedure to follow, once you deal with the strangeness of talking out loud to yourself.

I begin by setting up an audio recorder in a quiet location. Once the machine is recording, I tell the writer what I am going to do, e.g., "Louis, I'm about to read your second draft of your progress report dated May 27." Then I begin reading out loud. I verbalize everything, every word and thought, that comes to me as I go through the text. If making meaning of the text comes easily, then most of what is recorded is just vocalization of text. However, if I run into difficulty in making meaning, then much of what I verbalize is mental processing ... my different attempts to understand what the writer meant. (For an example, see Transcript \#1, line 7, "OK, now" to line 13, "represent a policy.") I don't mention spelling, punctuation or grammatical errors unless they interfere with my comprehension.

My basic stance is that of an average English-speaking reader; however, when reading a text which requires some specific action on the part of the reader, I put myself in the place of that particular audience in order to see if the information provided by the writer actually helps the reader accomplish the task. A specific instance: the writing task was to respond to a project officer's request for information regarding training courses for some of her employees. The average English-speaking reader would have been able to make sense of the text; however, when I tried to use the information as the project officer would have needed to use it, I found that the written response was not helpful at all because of the way in which the information was presented.

Besides reading through and making sense of the text, I also summarize what I understand the meaning to be. If the text is relatively short or of a general nature, I do this at the end. If the text is lengthy or detailed, then I do it at the end of each paragraph or section. (For an example of a final summary, see Transcript \#1, line 31 , "so it seems to me" to the end of the transcript.)

\section{How students use their tapes}

Students listen to their tapes while following the draft until the spoken message is clear. During this time, they often start editing their papers and inserting ideas/phrases in the margins. They often discuss concerns with me at this point, which leads naturally into explicit talk about the writing process. Then, when they are ready they prepare the next draft of their text. Both successive drafts and think-alouds are saved until the task is done so that students may verify changes and see progress. 


\section{Potential of the procedure}

Perhaps most important is that students get extensive, non-judgmental feedback on what they have written. That is, the teacher's verbalization of his or her thought processes highlights the creation of meaning and the dialogue between writer and reader rather than the grammatical accuracy of the written form.

The second point is that the student texts are not 'violated' as there is no need to write anything on them; thus, the students' sense of ownership of their texts is maintained.

Third: in reading a text, one may encounter problems in drawing meaning from the words in print. A frequent strategy at such a point is to experiment, not always successfully, with possible meanings to find one that fits both the language and meaning contexts. The think-aloud records this process on tape and thus provides the student with both a number of possible meanings and different ways of expressing them. This has three outcomes. One, I rarely talk explicitly about writing itself because the difficulties experienced (and recorded) in making sense of the text make lapses self-evident. For example, when I experience difficulty in making sense of an early part of the text and find my problem resolved later on, it tells the writer something about how the text is organized ... and probably in a more meaningful way than if, as a teacher, I said "I think this paragraph (or sentence) needs to come before that one." Two, listening to the reader/teacher negotiating the meaning from the text often helps students discover exactly what it is they want to say. (For an example, see Transcript \#1, line 15, "there is an alarm" to line 22, "furniture.") Three, the reader/teacher verbalizes different language exponents in making meaning of the text. These expressions provide students with models of language which they may choose to use as is or in modified form.

The final advantages of this procedure: it provides listening and speaking practice in an activity that is traditionally non-vocal, and it is not difficult to do; in fact, it is a pleasure to react to someone's writing without having to evaluate it.

\section{Conclusion}

If you are looking for a procedure which highlights the negotiation of discovery and meaning between writer and reader rather than grammatical correctness, my experience leads me to affirm the use of the think-aloud protocol. 


\section{APPENDIX}

Transcript \#1: think-aloud (with student's pre-think-aloud and postthink-aloud drafts)

\section{First draft: Pre-think-aloud}

Subject: Policy for Evacuation of Handicapped

In order that handicapped people evacuate safely out of the (name of building) when there is an alarm, and more specifically the handicapped on the 8th floor of the East Tower, a policy should:

- inform each of them that they have to leave when there is an alarm;

- know where the elevators are in such cases;

- head toward a designated elevator the minute they ear an alarm;

- go right ahead toward said elevator without panicking.

Note: Normal typeface is verbalization of thought processes; italic typeface is verbalization of the text.

1. OK, Louis, I'm reading your first draft of the 'policy for

2. evacuation of the handicapped' and I think I said that I wanted you

3. to do it specifically . . . to focus specifically on what the

4. handicapped in the East Tower, 8th floor should do. . . . In order

5. (read text through to line 8) the minute they ear an alarm . . .

6. they hear an alarm, it must be ... they hear an alarm . . go right

7. ahead toward said elevator without panicking. OK now, is this

8. the policy or is it saying what the policy is about . . . it seems

9. to me that the subject says policy for evacuation of

10. handicapped and yet is says that the policy should do this. In

11. other words . . . don't see this . . . as . . . a draft of the actual

12. policy ... um . . OK . . . so . . but there are some items that do

13. seem to represent a policy . . other than inform . . inform each

14. of them . . OK . . . each handicapped person should . . . leave when

15. there is an alarm . . . do they leave when there is . . an

16. intermittent alarm . . . or do they leave when there's the . . um

17. . . . continuous alarm . . they should know where the elevators are

18. in such cases . . . but where are the elevators . . again, if this

19. is going to be a policy, then they need to know where to go . . . go

20 . to the . . the . . um . . elevators that are used by employees or

21 . is it the . . um . . . the elevator that's used for moving stock and

22. furniture ... um . . OK, they should go towards the elevator the 
23. minute they hear an alarm . . . again, what kind of alarm . . .'cause

24. this statement really is very similar to leave when there is an

25. alarm, but leave doesn't tell them how to leave . . um . . go

26. right ahead toward said elevator without panicking . . . O.K. . . .

27. head toward . . . elevator is the same as go right ahead toward

28. . . elevator . . . so the only new information here is without

29. panicking . . . um . . . so . . . they've got to the elevator but they

30. still aren't out of the building . . . and that's an important part

31. of the policy . . . so it seems to me as a reader that I'm not sure

32. . . . whether or not this is the policy . . . there are some

33. guidelines . . . that is, as a handicapped person . . . whenever I

34. hear an alarm of any kind . . . um . . . I'm supposed to go to the

35. elevator without panicking . . . I'm not sure which elevator . . . and

36. I don't know what to do after that ...

Second draft: Post-think-aloud

(June 8, 1987)

Subject: Policy for Evacuation of Handicapped

Handicapped on the 8th floor of the (name of building) East Tower should:

- be informed of two kinds of alarms:

- slow beat alarm

- fast beat alarm or emergency alarm

If a fast beat or emergency alarm is heard:

- stop working;

- go to the closest elevator without panicking;

- go down to the main floor using any elevator;

- once on the main floor, go outside as fast as possible;

- do not wait for any designated person since there may be none;

- follow all instructions as they are given to other people;

- finally use your handicapped common sense to make sure you are safe!

If a slow beat alarm is heard:

- stay in your office;

- wait for instructions;

- relax! 


\section{NOTES}

1. For more information on the rationale behind the think-aloud protocol, see Afflerbach and Johnston (1984) and Hayes and Flower (1983).

\section{REFERENCES}

Afflerbach, P. \& Johnston, P. (1984). Research methodology: On the use of verbal reports in reading research. Journal of Reading Behaviour, XVI (4), 307-321.

Chenoweth, N.A. (1987). The need to teach rewriting. ELT Journal, 41 (1), 25-29.

Ericsson, K. and Simon, H. (1984). Protocol Analysis: Verbal reports as data. Cambridge, Mass.: MIT Press.

Flower, S.J. \& Hayes, J.R. (1984). A cognitive process theory of writing. Composition and Communication, 32 (4), 365-387.

Hayes, J.R. \& Flower, S.J. (1983). Uncovering cognitive processes in writing: An introduction to protocol analysis. In P. Mosenthal, L. Tamor, \& S.A. Walmsley (Eds.), Research on Writing: Principles and methods. New York: Longman, 206-219.

Marr, M. (1983). Verbal reports: How readers process unfamiliar text. Paper presented at the annual meeting of AERA, Montreal, April, 1983.

Raimes, A. (1985). What unskilled ESL students do as they write: A classroom study of composing. TESOL Quarterly, 19 (2), 229-252.

Zamel, V. (1985). Responding to student writing. TESOL Quarterly, 19 (1), 79101.

\section{THE AUTHOR}

Lynn McAlpine has considerable experience as a teacher of ESL adult students, as a materials developer, and as a trainer. She is intrigued with finding ways of enabling learners to have more control over their learning experiences. 